\title{
Brecha Digital
}

Regional:

Una oportunidad

para aliar esfuerzos

DOI: 10.29236/sistemas.n152a6

\section{Resumen}

En un contexto de vertiginosos cambios digitales se torna indispensable reflexionar y actuar al respecto de la equidad en términos de la tecnología. El presente artículo presenta un análisis bibliográfico con las principales tendencias y una propuesta para considerar aspectos para reducir la brecha digital en países como Colombia, y de esta manera desarrollar habilidades y competencias que le apuesten a un cambio social e inclusivo.

\section{Palabras clave}

Brecha digital, TIC, metodología

Julia Diaz, Catalina Ramírez, Diana Duarte, Luis Camilo Caicedo, Laura Guzmán, Luisa Payan, Janneth Arias, Nicolas Pacheco, Juan Pablo Ríos, Diego Martínez

\section{Introducción}

La brecha digital es un término asociado al analfabetismo digital. Va- rios actores se encuentran interesados en reducir esta brecha y analfabetismo, de un lado las empresas TIC que ofrecen productos y 
servicios y que buscan su máximo uso y de otro lado el sector público que tiene a su cargo proveer las condiciones para que los ciudadanos hagan uso de las TIC para su propio beneficio y desarrollo. En este contexto es fundamental revisar diferentes formas en las que se ha definido la brecha digital para tener una idea más concreta de lo que significa. Múltiples aproximaciones muestran que la brecha no es unidimensional y contar con dicho marco definitorio es el punto de partida para plantear cuales son los aspectos específicos que permitirían abordar un cierre de la brecha digital para un país en desarrollo como es el caso colombiano.

\section{¿Por qué la brecha digital y el analfabetismo digital son una preocupación para múltiples actores?}

El concepto de brecha digital se ha venido explorando por diferentes actores incluyendo instituciones públicas, organizaciones privadas, instancias educativas, entre otras. Se podría decir que muy particularmente autores de Norteamérica y Reino Unido iniciaron la investigación asociada a las situaciones derivadas por las diferencias que se presentan debido al acceso a las nuevas posibilidades de los computadores y el World Wide Web y aquellas que aún no utilizaban estas tecnologías. Factores como la ubicación geográfica, etnia, género, nivel educativo e ingresos fueron identificados como fuertes con- dicionantes para acceder a computadores e Internet en los años noventa '(Katz \& Aspden, 1997). Por tal motivo, diversos gobiernos iniciaron a hacer énfasis en el cómo lograr integrar la tecnología de información y comunicación a la reducción de brechas sociales (Kolko, 2002). Sin embargo, el concepto de brecha digital se limitaba a conocer si se tenía o no posesión de computadores y/o acceso al Internet, por lo que fue un término que recibió muchas críticas en círculos académicos, donde en repetidas ocasiones se ha buscado redefinir las implicaciones reales de la brecha digital como concepto (Comunello, 2010). Adicionalmente, es importante resaltar que la relación entre la brecha digital y los factores sociales, culturales y económicos ha sido ampliamente estudiada, demostrando que existe relación entre variables como el género, la edad, los ingresos económicos, la ruralidad, entre otros factores, y la brecha digital. Otros autores han estudiado la relación de la brecha digital con las desigualdades en los ingresos (Tirado-Morueta etal. 2017; Hilbert, 2010; Bauer, 2018), el género (Mumporeze \& Prieler 2017), el comportamiento según la situación socioeconómica (Rouwette etal. 1982), la etnia (Bartikowski etal. 2018), por mencionar tan solo algunos. Incluso en Colombia se ha estudiado la relación entre la brecha digital y los ingresos económicos o el género (Secretaría Distrital de Planeación de Bogotá 2014). 
Aproximación al significado de brecha digital regional

Dependiendo del autor, la Brecha Digital Regional podría abordarse desde diversas dimensiones. Por ejemplo, Van Dijk (2006) plantea cuatro tipos de acceso que inciden sobre el proceso de apropiación de una tecnología digital: acceso motivacional, acceso material, acceso por habilidades y acceso por usos. En ese sentido se podría decir que el acceso trasciende lo físico o referente al despliegue de infraestructura, pues es un proceso que abarca factores sociales, mentales y tecnológicos (Bucy \& Newhagen, 2004). En otras palabras, el acceso también incluye: las decisiones personales, influenciadas por el contexto social, que inciden sobre la adopción tecnológica; el aprendizaje de las competencias y habilidades necesarias para utilizar una tecnología; y el uso significativo que se da a la misma (Van Dijk, 2017). Así, basados en la teoría de la apropiación tecnológica de Van Dijk, la definición de apropiación de las TIC (MinTIC, 2017a), y la información primaria recolectada desde la comprensión de diversos actores del Sistema TIC, se podría definir que la brecha digital regional consta de 4 dimensiones: motivación, acceso material, habilidades digitales y aprovechamiento.

La motivación: son las percepciones de los individuos que determinan si ellos quieren, o no, tener algún tipo de interacción con las TIC, dado diversos factores. Por ejemplo: tecnofobia, la valoración social desfavorable de las TIC, una percepción de utilidad limitada, desconocimiento, entre otros. El Acceso Material: es la disponibilidad de los dispositivos tecnológicos (terminales) y de aquellos servicios e infraestructura (canales) necesarios para necesarios para la conexión y el uso de las funcionalidades de las TIC. Por ejemplo: la disponibilidad de una suscripción a Internet, software, servicio continuo de conexión eléctrica, entre otros. Las Habilidades Digitales son las cualificaciones necesarias para operar y/o usar las tecnologías. Estas se pueden dividir en diferentes niveles de habilidades, como, por ejemplo: la manipulación física adecuada; buscar, clasificar y seleccionar la información; crear, editar y hacer contribuciones a un medio digital; entre otros. Y el Aprovechamiento representado en los beneficios y la utilidad que se obtienen de las TIC en la vida cotidiana. Se puede abordar desde la intensidad, frecuencia y diversidad en los propósitos de uso de las tecnologías (comunicación, información, contenidos, entretenimiento, formación, transacciones, salud y trabajo).

\section{Las TIC para reducir la brecha digital regional}

La brecha digital regional puede ser explorada como resultado de las exclusiones sociales. Lo anterior puede afectar el crecimiento y el desarrollo debido a que la difusión 
de la tecnología puede favorecer la mano de obra cualificada y la creación de conocimiento "__"'" (Sidorenko \& Findlay, 2001). Un ejemplo es en el contexto de la educación. No hay evidencia suficiente para decir que la inversión en TIC mejore necesariamente el rendimiento de los estudiantes. Incluso, algunos de esos estudios concluyen que más allá del acceso a las TIC, los cambios en el rendimiento pueden estar asociados a la forma de apropiación y utilización que los profesores les den a estas herramientas en las aulas de clases.

Finalmente, otro aspecto relevante que puede ser afectado por la brecha digital es la participación ciudadana e inclusión social. Por ejemplo, estudios como los de Sylvester \& McGlynn (2010) concluyen que las personas que utilizaron Internet con más frecuencia en sus hogares eran más propensas a ponerse en contacto con el gobierno, ya fuera por teléfono, correo electrónico o carta. No obstante, esta participación no necesariamente está ligada al contacto con el gobierno sino también a la de su entorno cercano y comunidad. Por ejemplo, Alam \& Imran (Alam \& Imran, 2015) evidencian que el Internet de banda ancha puede considerarse como un medio eficaz de comunicación, intercambio de información, interacción y asimilación en la comunidad en general; para apoyar el desarrollo social e inclusión de los inmigrantes en Australia.
Conclusiones de un análisis metodológico para la reducción de la Brecha Digital Regional

La gran mayoría de los estudios encontrados presentan enfoques diferenciales importantes desde variables sociodemográficas como el sexo, la edad, el nivel educativo, el nivel de ingresos y la ocupación. En un caso se realizó un análisis de las condiciones de brecha digital a partir del uso de microdatos de encuestas nacionales (Toudert, 2015). Allí se hizo una agrupación regional de los microdatos, con el fin de comparar el desempeño de cada uno de los estados federados. Sin embargo, el trabajo central estuvo orientado a la creación de perfiles-tipo, con el fin de entender qué características describían mejor a los ciudadanos. Con base en 29 preguntas, principalmente asociadas a acceso material y aprovechamiento, se identificó que la mayoría de la población mexicana estaba asociada a perfiles marginados a las TIC.

Si bien es necesario reconocer que el enfoque regional puede omitir, e incluso distorsionar, algunos factores asociados a la brecha digital, sí permite entender a nivel nacional las diferencias entre sus diversas regiones, y que esto implica que las iniciativas deben estar pensadas desde el contexto local y de los intereses propios de la comunidad. Es importante, además, resaltar que un análisis regional debe estar 
acompañado de la interacción con otras variables de interés descritas anteriormente, como sexo, edad, estrato socioeconómico, educación y situación laboral.

Basado en las reflexiones presente artículo se propone que, considerando los diversos aspectos asociados a la brecha, se puedan articular efectivamente para generar un índice de brecha digital que permita establecer en estado de las regiones. La posibilidad de generar mediciones mediante la articulación de estos aspectos permitiría que las regiones comprendan su nivel de digitalización y las oportunidades de aprendizaje al timar acciones para reducir dicha brecha. El reto sería generar una metodología donde los actores del sector público y privado hicieran sinergia para generar mayor disminución de dicha brecha y por ende más oportunidades para las regiones

\section{Referencias}

Alam, K., \& Imran, S. (2015). The digital divide and social inclusion among refugee migrants. Information Technology \& People TA - TT -, 28(2), 344-365. Doi: http://doi.org/10.1108/ITP-04-20140083 LK -

https://univdelosandes.on.worldcat.org loclc/7054574732

Bauer, J.M. (2018). The Internet and income inequality: Socio-economic challenges in a hyperconnected society. Telecommunications Policy, 42(4), pp.333343. Available at:

http://dx.doi.org/10.1016/j.telpol.2017.0 5.009 .
Bartikowski, B. et al. (2018). The type-ofinternet-access digital divide and the well-being of ethnic minority and majority consumers: A multi-country investigation. Journal of Business Research, 82 (May 2017), pp.373-380.

Bucy, E., \& Newhagen, J. (2004). Media Access: Social and Psychological Dimensions of New Technology Use. London: LEA.

Comunello, F. (2010). From the Digital Divide to Multiple Divides: Technology, Society, and New Media Skills. En Handbook of Research on Overcoming Digital Divides: Constructing an Equitable and Competitive Information Society.

Hilbert, M. (2010). When is cheap, cheap enough to bridge the digital divide? Modeling income related structural challenges of technology diffusion in Latin America. World Development, 38(5), pp.756-770. Recuperado de:

http://dx.doi.org/10.1016/j.worlddev.20 09.11.019.

Katz, J. \& Aspden, P. (1997). Motivations for and barriers to Internet usage: results of a national public opinion survey. Internet Research, 7(3), pp.170-188.

Kolko, B.E. (2002). International IT Implementation Projects : Policy and Cultural Considerations. Reflections on Communication, pp.352-359.

Mumporeze, N. \& Prieler, M. (2017). Gender digital divide in Rwanda: A qualitative analysis of socioeconomic factors. Telematics and Informatics, 34(7), pp.1285-1293. Doi:

https://doi.org/10.1016/j.tele.2017.05.0 14.

Rouwette, E.A.J.A. et al. (1982). Modeling as persuasion: the impact of group model building on attitudes and behavior. Built Environment, 8(4), pp.267-271. 
Sidorenko, A., \& Findlay, C. (2001). The Digital Divide in East Asia. Asian-Pacific Economic Literature, 15(2), 18-30. Doi: http://doi.org/10.1111/1467-8411.00101

Secretaría Distrital de Planeación de Bogotá (2014). TIC y brecha digital: actualización de resultados 2014 ,

Recuperado de:

http://www.sdp.gov.co/sites/default/file s/2._tic_brecha_digital_2016.pdf

Sylvester, D. E., \& Mcglynn, A. J. (2010). The Digital Divide, Political Participation, and Place. Social Science Computer Review, 28(1), 64-74.

Doi:http://doi.org/10.1177/.

MinTIC. (2017a). Glosario. Recuperado el 27 de abril de 2018, a partir de

http://www.mintic.gov.co/portal/604/w3propertyvalue-1051.html

MinTIC. (2017b). Primera gran encuesta TIC. Estudio de acceso, uso y retos de las TIC en Colombia.
Tirado-Morueta, R. et al. (2017). The Relativity of Sociodemographic Determinism on the Digital Divide in High School Students in Ecuador. International Journal of Communication, 11, pp.1528-1551. Available at: http://ijoc.org .

Toudert, D. (2015) Brecha digital y perfiles de uso de las tic en México: Un estudio exploratorio con microdatos. Revista Culturales, 11(1). 167-200.

Recuperado de:

http://www.redalyc.org/articulo.oa?id=6 9438994006

Van Dijk, J. (2006). Digital divide research, achievements and shortcomings. Poetics, 34(4-5), 221-235. Doi:

https://doi.org/10.1016/j.poetic.2006.05 .004

Van Dijk, J. (2017). Digital Divide: Impact of Access. The International Encyclopedia of Media Effects, 1-11. Doi: https://doi.org/10.1002/978111878376 4.wbieme0043 裂

Autores. Julia Diaz PhD Administración Universidad de los Andes, Catalina Ramírez PhD Ingegneria Gestionale Politecnico di Milano; Diana Duarte Magister Ingeniería Industrial Universidad de los Andes; Luis Camilo Caicedo Magíster Ingeniería Industrial Universidad de los Andes; Laura Guzmán Magister Ingeniería Industrial Universidad de los Andes; Janeth Arias Magister Antropología Universidad de los Andes; Luisa Payan Magister Desarrollo Regional Universidad de los Andes; Nicolas Pacheco Magister Ingeniería Industrial Universidad de los Andes; Juan Pablo Ríos Magister Ingeniería de Sistemas Universidad Nacional; Diego Martínez Magister Ingeniería Industrial Universidad Nacional. 